\title{
Mesure des caractéristiques mécaniques du polycarbonate sollicité en traction uniaxiale et en température par diffraction optique des réseaux
}

\author{
Lucien Meva'A ${ }^{1, a}$, Alexis Lagarde ${ }^{2}$ et Fabrice Brémand ${ }^{2}$ \\ 1 ENSP, BP 8390, Yaoundé, Cameroun \\ 2 Laboratoire de Mécanique des Solides, UMR 6610, Université de Poitiers, Bâtiment SP2MI, Boulevard Pierre \& Marie Curie, \\ BP 30179, 86962 Futuroscope Chasseneuil, France
}

Reçu le 29 avril 2002, accepté le 3 décembre 2004

\begin{abstract}
Résumé - Il existe en mécanique, de nombreuses méthodes de détermination des caractéristiques mécaniques des matériaux (jauges électriques, extensomètres mécaniques...). Les méthodes optiques apportent dans ce domaine un plus, notamment à cause de leur particularité d'être des méthodes de mesure sans contact. Cet article décrit la méthode de diffraction optique des réseaux appliquée à la détermination des caractéristiques mécaniques du polycarbonate, sollicité en traction uniaxiale en température pour des éprouvettes planes et à l'ambiante pour des éprouvettes cylindriques.
\end{abstract}

Mots clés : Réseaux / diffraction optique / traction uniaxiale / caractéristiques mécaniques / éprouvettes cylindriques

\begin{abstract}
Mechanical characteristic measurement of polycarbonate submitted to uniaxial tension in temperature by optical diffraction. Many methods to determine the characteristics of materials exist (mechanical extensometers, electrical gauges...). Optical methods bring something new in this domain because of their particularity of being non contact and non disturbing methods measurement. This article describes the optical diffraction of grid method for the measurement of the characteristics of polycarbonate in plane sample and in temperature on cylindrical specimen, both submitted to a uniaxial traction in temperature and on cylindrical specimen.
\end{abstract}

Key words: Grid / optical diffraction / uniaxial load / mechanical characteristic / cylindrical sample

\section{Introduction}

La méthode de diffraction optique des réseaux est une méthode de mesure sans contact des déformations basées sur l'analyse d'un réseau de traits marqués ou gravés à la surface d'une pièce $[1,2]$. Elle a déjà été utilisée pour la détermination des lois de comportement de divers matériaux, notamment certains polyuréthannes [3]. Les caractéristiques mécaniques sont obtenues par comparaison entre un état de référence et les différents états de chargement. Cette technique suppose que la déformation soit homogène dans la zone de mesure et que la surface de la pièce soit localement plane.

Après avoir rappelé le principe de la déformation d'un réseau de traits croisés marqué à la surface d'une éprouvette, nous présentons la méthode de diffraction optique des réseaux et son application sur des éprouvettes

\footnotetext{
a Auteur correspondant : lucien-mevaa@hotmail.com
}

de polycarbonate sollicitées en traction uniaxiale, à l'ambiante et en température.

\section{Déformation d'un réseau de traits croisés}

Le réseau de traits à la surface des éprouvettes est gravé par interférométrie laser [4]. Il est constitué pratiquement d'éléments élémentaires juxtaposés. Un élément, dans sa position initiale est défini par la position de quatre points $\mathrm{O}, \mathrm{A}^{\mathrm{i}}, \mathrm{B}^{\mathrm{i}}, \mathrm{C}^{\mathrm{i}}$. À l'état déformé, il se transforme en un parallélogramme $\mathrm{O}, \mathrm{A}^{\mathrm{d}}, \mathrm{B}^{\mathrm{d}}, \mathrm{C}^{\mathrm{d}}$ (Fig. 1). Le point $P(X, Y)$ dans la configuration initiale $\Gamma_{0}$ se transforme, dans la configuration actuelle $\Gamma_{(t)}$ en $m(x, y)$. Si $\overline{\bar{F}}$ est le tenseur gradient représentatif de la transformation, nous pouvons déterminer à partir de ses composantes, les composantes du tenseur de Cauchy-Green droit $\overline{\bar{C}}$ tel que $\overline{\bar{C}}={ }^{t} \overline{\bar{F}} \overline{\bar{F}}$. 

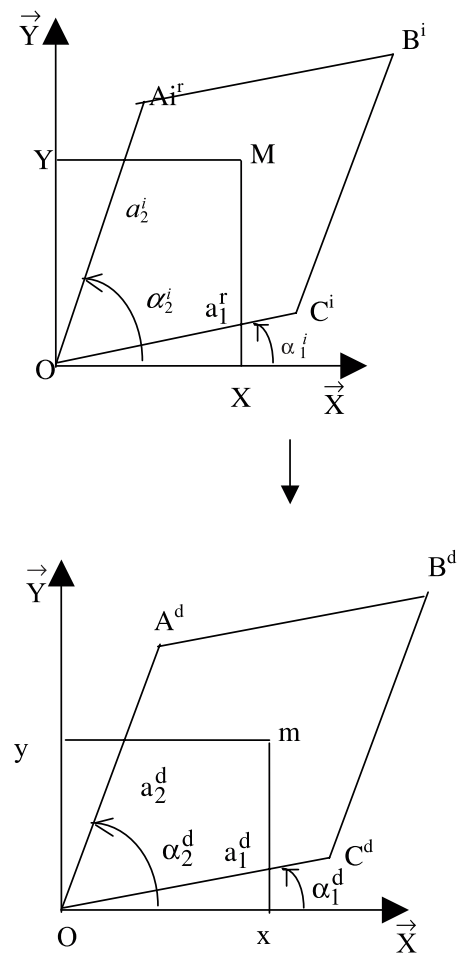

Fig. 1. Déformation d'un élément unitaire.

Connaissant la géométrie de l'état de référence, les composantes du tenseur des déformations ne sont fonction que des quatre paramètres géométriques définissant l'état déformé à savoir les deux orientations $\alpha_{1}^{\mathrm{d}}$ et $\alpha_{2}^{\mathrm{d}}$ ainsi que les deux distances $a_{1}^{\mathrm{d}}$ et $a_{2}^{\mathrm{d}}$. Comme ces deux distances sont difficiles à mesurer, pratiquement nous préférons considérer plusieurs éléments juxtaposés. Les paramètres à mesurer deviennent les pas $p_{1}$ et $p_{2}$ et les deux orientations $\alpha_{1}^{\mathrm{d}}$ et $\alpha_{2}^{\mathrm{d}}[5]$.

\section{Diffraction optique des réseaux}

\subsection{Mise en évidence du phénomène}

La diffraction optique d'un faisceau de lumière cohérente et parallèle de longueur d'onde $\lambda$ par un réseau de pas $p$ est un phénomène bien connu. L'étude est faite soit en réflexion, soit en transmission. La figure obtenue est constituée de taches ponctuelles et circulaires représentant chacun un ordre diffracté. Elle est observée sur un écran placé à la distance $L$ du réseau de traits. Chaque famille de tache de diffraction est perpendiculaire à la direction des traits du réseau qui l'ont généré (Fig. 2). Cela permet d'obtenir l'orientation des familles de traits du réseau. Dans le cas de la diffraction à l'infini, les distances entre les taches sont inversement proportionnelles au pas du réseau. Les relations classiques de la diffraction sont données par les équations suivantes :

- en transmission :

$$
\sin \theta_{k}-\sin \theta_{0}=\frac{k \lambda}{p}
$$

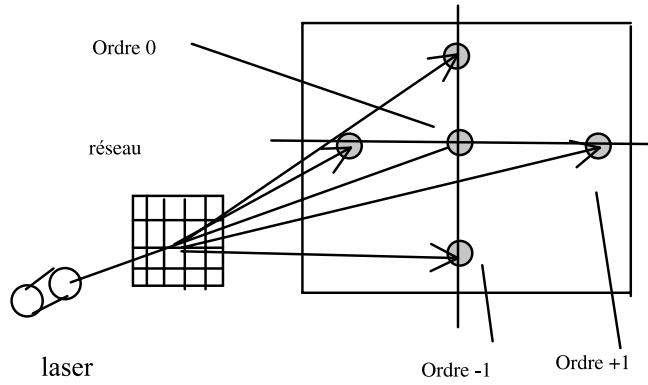

Fig. 2. Réseau et sa figure de diffraction.

- en réflexion :

$$
\sin \theta_{k}+\sin \theta_{0}=\frac{k \lambda}{p}
$$

$\theta_{k}$ est l'angle de diffraction d'ordre $k$ et $\theta_{0}$ est l'angle d'incidence du faisceau laser.

\subsection{Principe de l'analyse des figures}

L'utilisation d'un système d'acquisition et de traitement d'image permet d'automatiser et d'améliorer la rapidité dans le dépouillement et l'interprétation des figures de diffraction [6]. Pour chaque état de charge, les images correspondantes sont enregistrées à l'aide d'une caméra CCD et stockées en mémoire. L'analyse est effectuée automatiquement par la recherche du centre géométrique des taches de diffraction d'ordre $0,+1$ et -1 .

À chaque figure de diffraction correspond un état de charge donné. On définit une zone rectangulaire autour de chacune des taches dans laquelle le programme d'analyse recherche sa position $\left(x_{\mathrm{g}}, y_{\mathrm{g}}\right)$. La technique d'interpolation utilisée repose sur le calcul du barycentre pondéré par les intensités lumineuses. On a alors :

$$
x_{\mathrm{g}}=\frac{\sum_{i} x_{i}\left(I_{i}-I_{\mathrm{s}}\right)}{\sum_{i} I_{i}}
$$

et

$$
y_{\mathrm{g}}=\frac{\sum_{i} y_{i}\left(I_{i}-I_{\mathrm{S}}\right)}{\sum_{i} I_{i}}
$$

où $\left(x_{i}, y_{i}\right)$ sont les coordonnées du pixel $i$ d'intensité lumineuse $I_{\mathrm{s}}, I_{\mathrm{S}}$ une intensité seuil permettant d'éliminer le bruit de fond. Connaissant la position des taches dans chaque fenêtre, nous pouvons calculer les distances $d_{1}$ et $d_{2}$ entre la paire de taches horizontales d'ordre +1 et -1 ainsi que les orientations $\beta_{1}$ et $\beta_{2}$ (Fig. 3) par rapport à l'horizontale de la grille de la caméra CCD utilisée pour filmer les images.

Par la suite, le calcul des pas $p_{1}$ et $p_{2}$ du réseau déformé s'obtient à l'aide de la relation classique de la diffraction. Dans le cas pratique d'un faisceau laser incident 


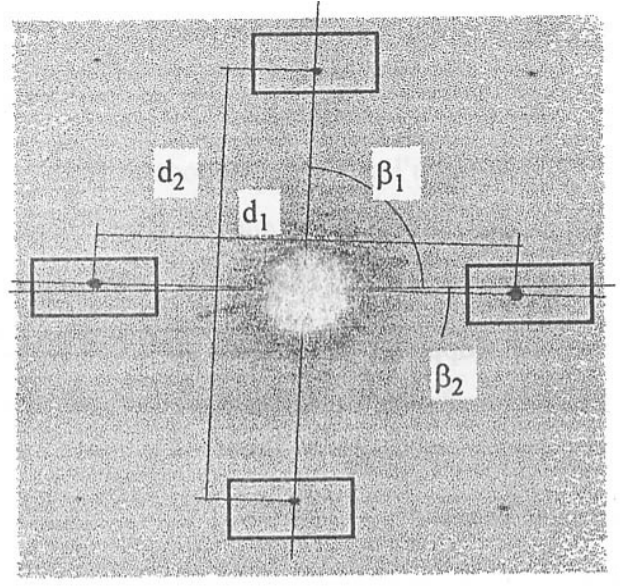

Fig. 3. Ordres diffractés.

normal à la surface de l'éprouvette, nous obtenons les relations suivantes :

$$
\begin{aligned}
p_{1} & =\frac{\lambda}{\sin \left(\operatorname{Arctg}\left(\frac{d_{1}}{2 L}\right)\right)} \\
p_{2} & =\frac{\lambda}{\sin \left(\operatorname{Arctg}\left(\frac{d_{2}}{2 L}\right)\right)}
\end{aligned}
$$

Les inclinaisons de chaque réseau sont données par :

$$
\alpha_{1}=\beta_{1}+\frac{\pi}{2}
$$

et

$$
\alpha_{2}=\beta_{2}+\frac{\pi}{2}
$$

\section{Expérimentation}

\subsection{Gravure des réseaux de diffraction}

Avant chaque gravure, il est nécessaire de polir la surfaces des pièces. Le but visé est d'obtenir une zone où sera gravée le réseau, exempt de toutes les rayures qui sont susceptibles de donner des taches de diffraction inexploitables. Par la suite, la gravure du réseau de traits équidistants se fait par interférométrie laser. Cette technique est basée sur le principe de franges d'interférence de deux faisceaux issus d'une même source laser pulsé néodyme à Yag capable d'atteindre une énergie maximale de 1 joule par tir, lors d'impulsions monomodes de brèves durées (15 ns) et de fréquences variant de 0,05 à $10 \mathrm{~Hz}$. Les franges sont obtenues en champ parallèle, soit directement, soit à partir du rayon issu du laser en pleine puissance, soit après avoir étendu le faisceau avec un système de lentilles de même point focal. Expérimentalement, au niveau de la surface de l'éprouvette à graver, le champ d'interférence est réalisé en combinant le faisceau issu directement de la source avec celui réfléchi par un miroir

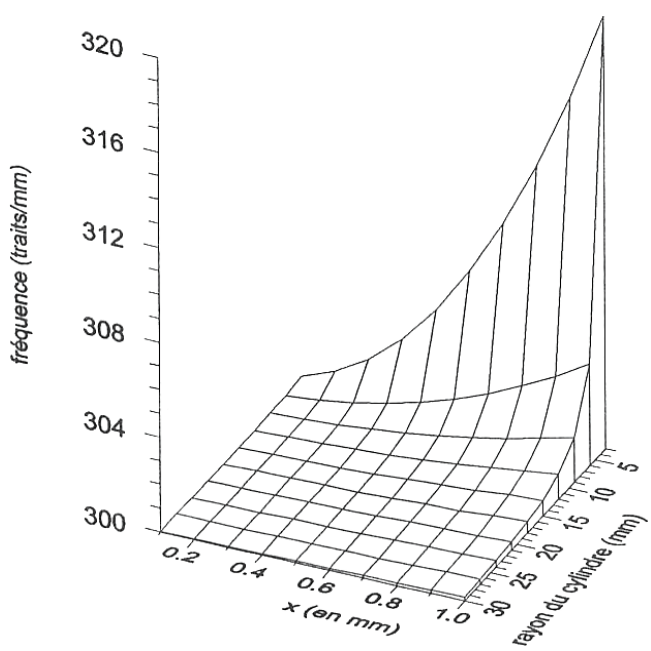

Fig. 4. Variation de la fréquence en fonction du rayon.

orienté à $90^{\circ}$ de cette surface. La fréquence du réseau pour une surface plane est donnée par la relation :

$$
F_{0}=\frac{2 \sin \alpha}{\lambda}
$$

Pour une surface cylindrique, le réseau dont les traits sont gravés suivant la courbure de la surface ont comme fréquence, la fréquence $F_{0}$. Pour le réseau dont les traits sont perpendiculaires à la courbure, la fréquence en un point donné $x$ de la surface varie avec le rayon $R$ (Fig. 4) suivant la relation :

$$
F_{0}=\frac{R}{P_{0}} \frac{\lambda}{\sqrt{R^{2}-x^{2}}}
$$

Les réseaux orthogonaux sont obtenus par rotation de la pièce autour d'un axe perpendiculaire à la surface à graver.

\subsection{Résultats expérimentaux sur éprouvettes planes}

Les éprouvettes planes de polycarbonate une fois gravées, sont placées dans une étuve couplée à un régulateur de température qui permet d'imposer le cycle de température voulu. Il est important de bien positionner l'éprouvette entre les mors du système de traction et de bien régler la précharge afin d'éviter que la ventilation d'homogénéisation de la température ne provoque des déplacements hors plan auxquels est sensible la méthode de diffraction optique. Nous nous sommes fixés sept cycles de température de $40^{\circ}$ à la température de transition vitreuse du polycarbonate qui est de $148,9^{\circ}$. Les mesures sont réalisées sur un palier de température qui dure $1 \mathrm{~h} 30 \mathrm{~min}$, ce qui est suffisant pour avoir plusieurs états de charge. Les figures 5 à 11 nous montrent les courbes contraintes-déformations relatives aux différentes températures d'essai.

La méthode de diffraction optique des réseaux nous permet d'obtenir simultanément les déformations suivant les deux directions. Par la suite, nous déduisons 


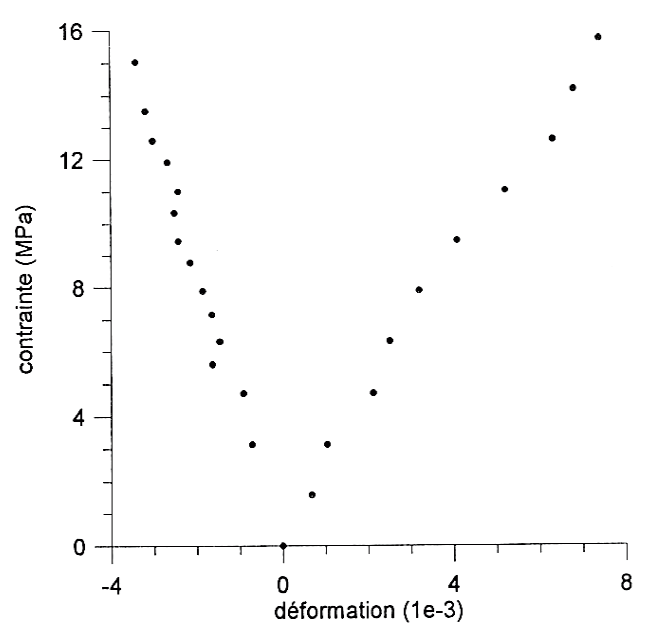

Fig. 5. Courbe à $40{ }^{\circ} \mathrm{C}$.

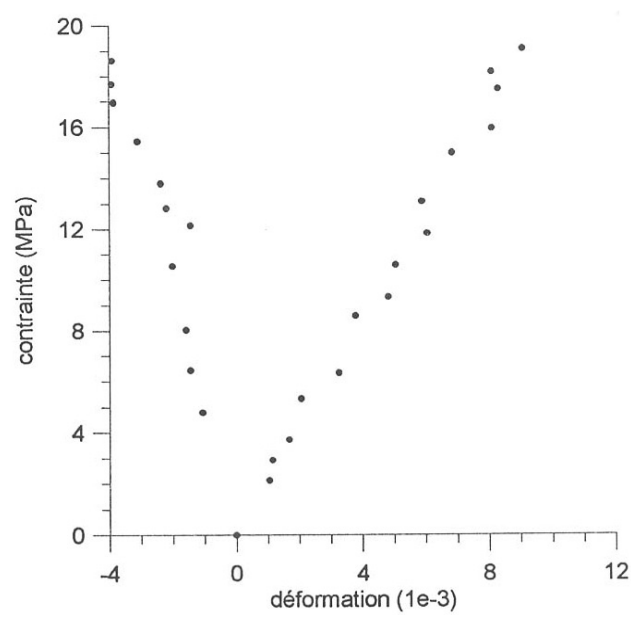

Fig. 7. Courbe à $80^{\circ} \mathrm{C}$.

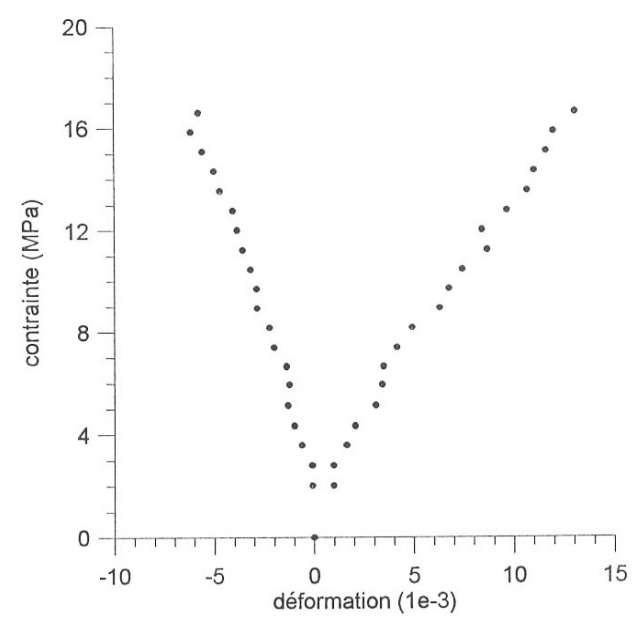

Fig. 9. Courbe à $120^{\circ} \mathrm{C}$.

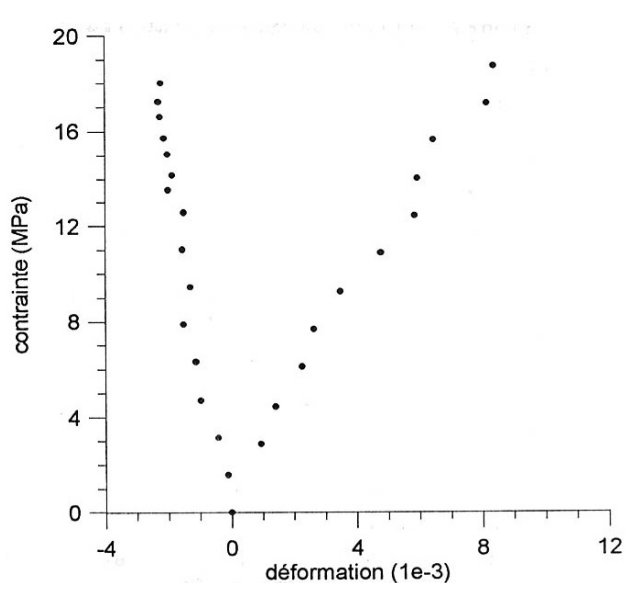

Fig. 6. Courbe à $60^{\circ} \mathrm{C}$.

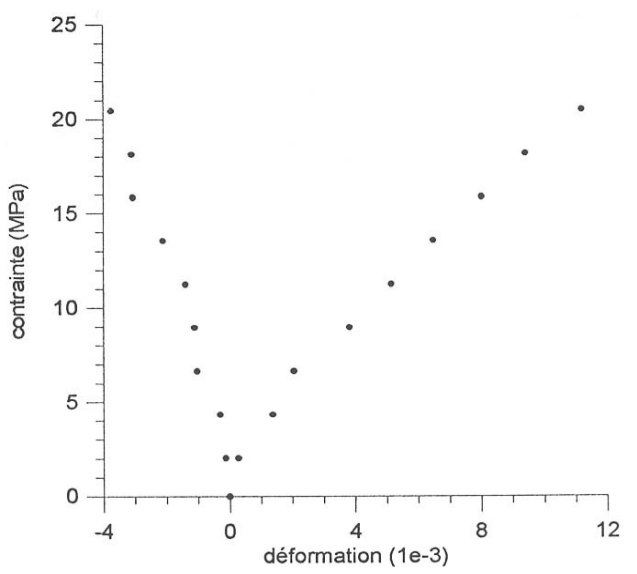

Fig. 8. Courbe à $100{ }^{\circ} \mathrm{C}$.

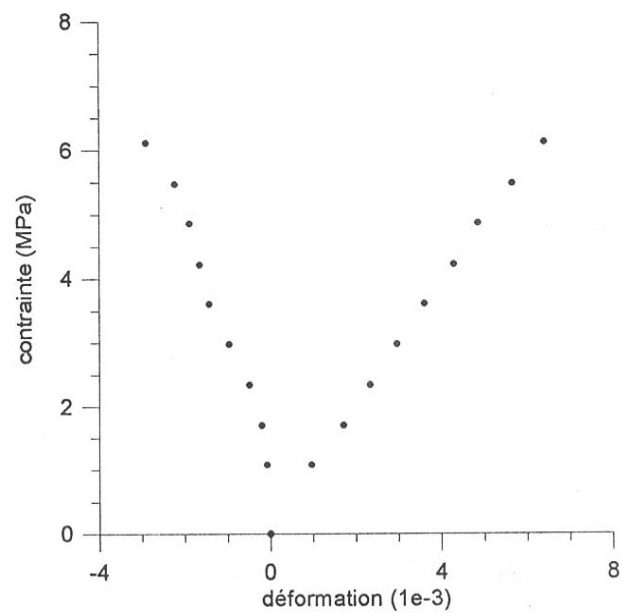

Fig. 10. Courbe à $140{ }^{\circ} \mathrm{C}$. 


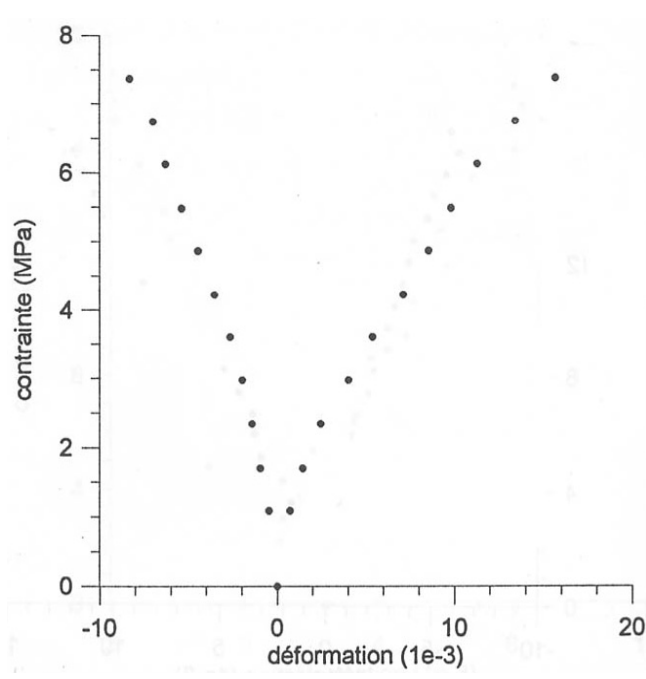

Fig. 11. Courbe à la température de figeage.

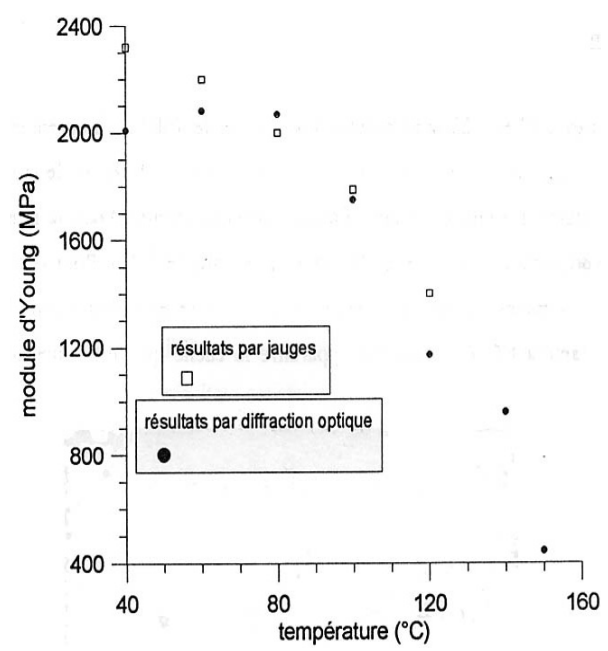

Fig. 12. Comparaison de la courbe module d'Youngtempérature, par diffraction optique et par les jauges électriques.

l'évolution du module d'Young en fonction de la température comparativement avec celle obtenue par Brinson et Caplan [7] qui utilisent des jauges électriques pour mesurer les déformations, des thermocouples et un thermomètre pour mesurer la température (Fig. 12).

La figure 12 nous montre une décroissance de la valeur du module d'Young avec la température. L'allure de la courbe obtenue par les deux méthodes est la même. L'erreur relative de la méthode de diffraction optique par rapport à la méthode utilisant les jauges est comprise entre $3 \%$ et $12 \%$. Cette erreur doit tenir compte de la dégradation de la qualité du réseau avec la charge et la température. Dans certains cas nous avons une fluctuation des points de déformation transversale de l'ordre de $5 \times 10^{-4}$, valeur qui n'est pas loin de la limite de résolution de la méthode de diffraction.

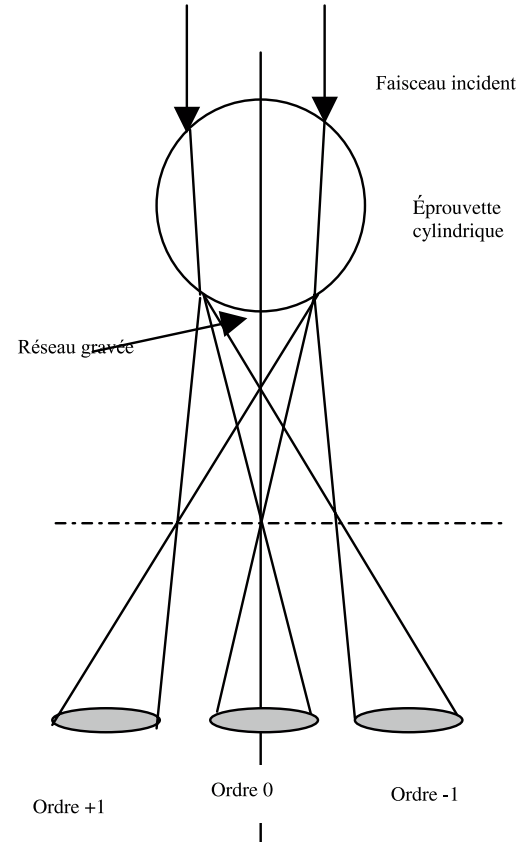

Fig. 13. Trajet des faisceaux diffractés par le réseau à pas variable.

\subsection{Résultats expérimentaux sur éprouvettes cylindriques}

Nous avons appliqué la méthode de diffraction optique des réseaux à la mesure des déformations sur des éprouvettes cylindriques de polycarbonate de diamètre $R=9 \mathrm{~mm}$ sollicitées en traction uniaxiale. Les éprouvettes cylindriques se comportent comme des lentilles cylindriques dont la focale est donnée par la relation :

$$
f=\frac{R}{2(n-1)}
$$

la valeur de l'indice $n$ de réfraction du polycarbonate est de 1,59. Pour un faisceau laser incident dont la direction de propagation est normale à l'axe de l'éprouvette et qui arrive sur sa face gravée, les ordres diffractés par le réseau à pas variable convergent sur un écran d'observation placé entre la gravure et le plan focal image et divergent lorsque l'écran est placé derrière le plan focal (Fig. 13). Pour le réseau à pas constant, l'éprouvette cylindrique se comporte par rapport au faisceau incident comme une éprouvette plane.

Comme les focales sont très faibles, et compte tenu de la fréquence expérimentale du réseau initial (300 traits.mm ${ }^{-1}$ ), il est difficile de placer un écran d'observation dans le plan focal où convergent les faisceaux diffractés. Lorsque l'écran n'est pas placé dans le plan focal, nous sommes confrontés au phénomène de l'étalement des taches de diffraction. Pour les réduire et les rendre exploitables, nous utilisons une lentille cylindrique de focale beaucoup plus grande que celle de l'éprouvette [8]. La base de mesure est alors très réduite $\left(1 / 100^{\mathrm{e}}\right.$ de $\left.\mathrm{mm}\right)$ et l'arc de cercle sur lequel est gravé le réseau peut être confondu avec la tangente à la surface du cylindre. Les faisceaux 


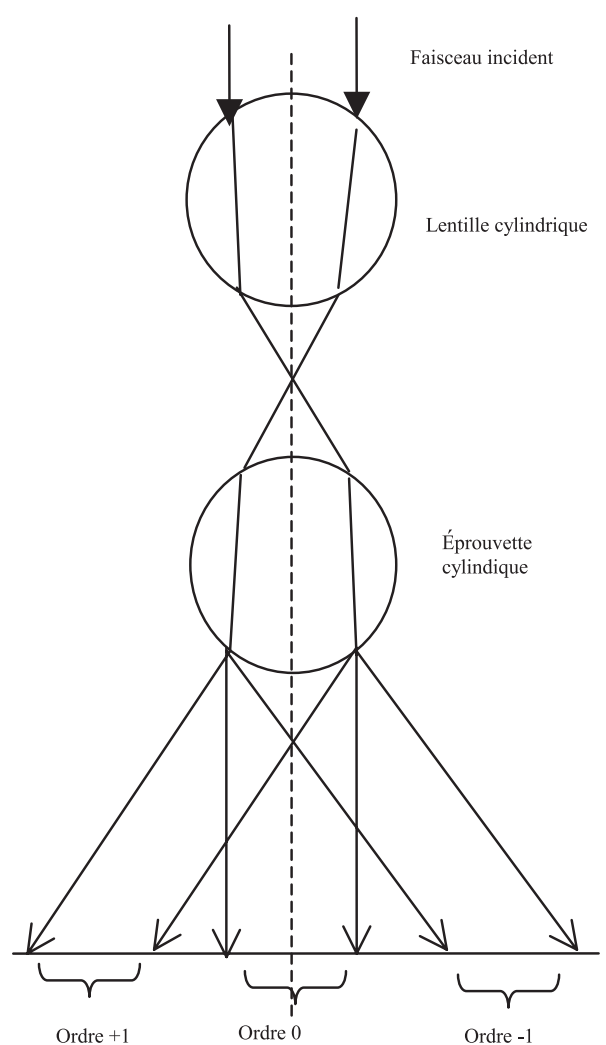

Fig. 14. Influence de la lentille cylindrique sur les faisceaux diffractés.

à la sortie du dispositif sont parallèles et les taches de diffraction circulaires (Fig. 14).

Une fois ce dispositif monté, nous sollicitons l'éprouvette en traction uniaxiale Dans la figure 15, nous comparons les déformations que nous avons obtenus sur les éprouvettes cylindriques avec celles obtenues sur les éprouvettes planes par la diffraction optique. Nous constatons que les courbes sont similaires. Le module d'Young et le coefficient de Poisson sont :

\begin{tabular}{lll}
\hline & $E(\mathrm{Mpa})$ & $\nu$ \\
\hline Éprouvette plane & 2236 & 0,4 \\
Éprouvette cylindrique & 2287 & 0,38 \\
\hline
\end{tabular}

Nous obtenons des valeurs qui sont similaires, les incertitudes relatives (de l'ordre de $2 \%$ pour $E$ et de $5 \%$ pour $\nu$ ) sont imputables à l'expérimentation (qualité du réseau notamment, dimension de la zone de mesure).

\section{Conclusion}

La méthode de mesure des déformations par diffraction optique des réseaux est une méthode de mesure sans contact. Comme nous le montre cet article, moyennant certaines conditions expérimentales liées à la qualité du réseau notamment, elle peut être utilisée en environnement hostile en température. Nous montrons également qu'elle peut être utilisée, en transmission, pour la mesure des déformations sur une surface cylindrique et les résultats obtenus démontre la pertinence et la fiabilité de cette méthode. Nous avons réalisé, à la fin de notre étude à des essais sur de l'acier à $1200^{\circ} \mathrm{C}$ par rapport à la qualité des taches de diffraction qui se sont avérés encourageants. Ce qui offre de nouvelles perspectives.

\section{Références}

[1] F. Brémand, Photoélasticité en grandes déformations, Méthodes de mesures de grandes et petites déformations, Thèse de doctorat de l'Université de Poitiers, 1988

[2] F. Brémand, A. Lagarde, Méthode optique de mesure des déformations utilisant le phénomène de diffraction, Compte-Rendu à l'Académie des Sciences II, 303, 1986, pp. $520-525$

[3] F. Brémand, J.C. Dupré, A. Lagarde, Non contact and non disturbing local strain measurements methods, Parts I, Principle, Eur. J. Mech. 11A (1992) 349-366

[4] B. Moreau, M. Cottron, A. Lagarde, Gravure par interférométrie laser de réseaux denses, Journées AMACGAMC, Caractérisation mécanique des composites, Saint-Étienne, 1989

[5] L. Meva'a, A. Lagarde, F. Brémand, Étude numérique de l'influence de la courbure des éprouvettes cylindriques de duralumin sollicitées en traction unixiale sur les déformations tangentielles par analyse spectrale des réseaux, Mécanique industrielle et matériaux, revue du GAMI 54 (1999) 142-145

[6] J.C. Dupré, Traitement et analyse d'image pour la mesure de grandeurs cinématiques, déplacements et déformations à partir de la granularité laser et de réseaux croisés, et pour l'étude de couplage thermomécanique, Thèse de doctorat de l'Université de Poitiers, 1992

[7] H.F. Brinson, E.S. Caplan, College of Engineering, Nonlinear viscoelastic Characterization of polycarbonate, Virginia Polytechnic Institute and State University, 1982

[8] L. Meva'a, F. Brémand, A. Lagarde, Optical methods for strain measurement of cylindrical specimens submitted to uniaxial load, 10th Conference on Experimental Mechanics, 18-22 July 1994, Lisbon 